\title{
Submillimetre wave 3D imaging radar for security applications
}

\author{
D.A. Robertson *, D.G. Macfarlane *, S.L. Cassidy *, T. Bryllert †, E. Gandini *, N. Llombart * \\ * University of St Andrews, St Andrews, Scotland,dar@st-and.ac.uk,dgm5@st-and.ac.uk, scott@scottlc.net \\ †Wasa Millimetre Wave AB, Gothenburg, Sweden, bryllert@wmmw.se \\ †Technical University of Delft,Delft, Netherlands,E.Gandini@tudelft.nl,N.LlombartJuan@tudelft.nl
}

Keywords: Submillimetre wave radar, security, FMCW, 3D imaging, high resolution.

\begin{abstract}
There is ongoing worldwide interest in finding solutions to enhance the security of civilians at airports, borders and high risk public areas in ways which are safe, ethical and streamlined. One promising approach is to use submillimetre wave $3 \mathrm{D}$ imaging radar to detect concealed threats as it offers the advantages of high volumetric resolution $(\sim 1 \mathrm{~cm} 3)$ with practically sized antennas $(<0.5 \mathrm{~m})$ such that even quite small objects can be resolved through clothing. The Millimetre Wave Group at the University of St Andrews has been developing submillimetre wave 3D imaging radars for security applications since 2007. A significant goal is to achieve near real-time frame rates of at least $10 \mathrm{~Hz}$, to cope with dynamic scenes, over wide fields of view at short range with high pixel counts. We review the radar systems we have developed at 340 and $220 \mathrm{GHz}$ and the underpinning technologies which we have employed to realise these goals.
\end{abstract}

\section{Introduction}

The ability to detect person-borne concealed threats is an ongoing and pressing requirement in many scenarios such as border crossings, airports and military checkpoints. Different scenarios require (or tolerate) different concepts of operation such as long-range stand-off investigation, virtual pat-downs within stop-and-pause portals, or short-range walk-by screening. Many different technologies have been developed and evaluated to meet these needs and each has their strengths and weaknesses and a combination of sensors may offer the best overall detection performance. Sensors operating in the millimetre and submillimetre wave parts of the spectrum are good candidates as they offer the advantages of adequate penetration through fabrics and high spatial resolution with systems of a practical size [1].

For applications in which a portal is appropriate, active millimetre wave body scanners based on cylindrically scanned synthetic aperture radar techniques have seen widespread deployment and considerable commercial success [2]. However, they are not without drawbacks and there is an ongoing requirement to improve detection performance and increase throughput. For long-range stand-off screening, at ranges of many to tens of metres, operation at shorter submillimetre wavelengths is required to achieve the necessary diffraction limited resolution with a practically sized antenna [3].

The Millimetre Wave Group at the University of St Andrews, in collaboration with various partners, has been developing submillimetre wave radar systems since 2007 which address both short- and long-range security applications. An overriding aim of our work has been to develop such imagers which cope with the real world dynamic nature of people so our technological developments have been driven strongly to achieve near real-time frame rates of at least $10 \mathrm{~Hz}$.

In this paper we review our work developing submillimetre wave 3D imaging radars for security applications and discuss the underlying technologies we have employed.

\section{Overview of submillimetre wave radar}

Most submillimetre wave radars make use of solid-state frequency multiplier chains [3, 4, 5, 6, 7], exploiting both MMIC and Schottky diode technologies, to generate transmit powers which are typically in the milliwatt class. When combined with sensitive heterodyne or homodyne receivers, these radars can achieve high dynamic ranges. Whilst solidstate power and low noise amplifier devices at these frequencies are constantly improving [8] they have yet to be adopted in submillimetre wave radar systems. Due to the low power, solid state transmitters, the most common radar technique employed is frequency modulated continuous wave (FMCW). The high carrier frequency enables the use of frequency chirps with a wide absolute bandwidth (yet still modest fractional bandwidth) which can yield very fine range resolutions e.g. $1 \mathrm{~cm}$ range bins for $15 \mathrm{GHz}$ chirp bandwidth. The challenge in achieving high fidelity radar measurements with such bandwidths is to achieve wideband chirps which have good linearity, low phase noise and short duration, especially if fast pixel rates are required [9]. This places demands on the chirp generator used to drive the multipliers, which is usually at microwave frequencies, and the spectral characteristics of all the components in the frequency multiplication chain (such as amplitude flatness and group delay variations), both of which contribute to distortion of the radar's point response [4, 7].

For effective security screening when searching for concealed objects, the radar must be capable of resolving those which are considered a threat and this usually implies that a spatial resolution or spot size of about $1 \mathrm{~cm}$ is required. From basic diffraction, operation at submillimetre wavelengths means 
this level of resolution can be achieved with practically sized antennas, approximately in the range 30 to $100 \mathrm{~cm}$, depending on the desired operational distance which may range from just a metre or two to many tens of metres, depending on the application. Either lenses or mirrors can be used, the latter offering negligible loss and back reflections but tending to lead to more bulky optical arrangements.

To be practical in real-world applications, submillimetre wave 3D imaging radars need frame rates commensurate with dynamic scenes and real people as subjects. Due to the cost and complexity of the transmitter and receiver electronics, such radars typically have just one or only a few channels so some form of beam scanning is required for image formation. To achieve frame rates of order several $\mathrm{Hz}$ then presents a challenge in how to achieve rapid beam steering. For long range applications in which the subtended angle of the field of view (FoV) may be quite limited, a successful approach can be to use small aperture (and hence rapid) beam steering optics behind a magnifying quasi-optical telescope of lenses or mirrors. However, the magnification $\mathrm{M}$ of the telescope requires that the beam steering deflection angle has to be bigger by a factor of $\mathrm{M}$. In short-range applications in which the FoV may be quite wide (e.g. if trying to image a whole person) this approach is not feasible and the beam steering optics will need to be placed at the entrance aperture of the imager. This then presents a challenge for the high speed mechanical movement of large aperture beam steering components. Ultimately, the radar designer has to consider a complex trade-off between spot size, range, FoV and frame rate.

\section{IRAD $340 \mathrm{GHz}$ radar}

We developed the IRAD $340 \mathrm{GHz}$ radar [6] for stand-off applications at $20+\mathrm{m}$ range. It uses a coherent heterodyne architecture with separate Schottky diode multiplier chains for transmit (x48) and local oscillator (x24) signals. A key objective with IRAD was to achieve high frame rates to enable the investigation of phenomenology of realistic scenes and targets. The system is capable of imaging at up to $10 \mathrm{~Hz}$ frame rate but this was achieved by trading off field of view which is a modest $0.5 \times 0.5 \mathrm{~m}$ at $20 \mathrm{~m}$ range and voxel resolution which is $3 \times 3 \times 4.2 \mathrm{~cm}^{3}$. IRAD transmits a single linear polarisation and receives both co- and cross-polar signals for subsequent analysis. Fig. 1 shows IRAD in operation.

As noted above, generating rapid, wideband chirps with low phase noise is crucial in submillimetre wave radars which employ significant frequency multiplication (phase noise degrades by $20 \log \mathrm{N}$ where $\mathrm{N}$ is the multiplication factor). Phase noise in the transmitted signal can be reflected from bright targets leading to a raised noise floor which can mask smaller targets in the same line of sight (e.g. objects concealed under layers of clothing) [10]. For IRAD we showed that upconverting a chirp from a direct digital synthesiser (DDS) onto a low noise stable local oscillator (STALO) yields can yield substantially lower phase noise (e.g. by $20 \mathrm{~dB}$ ) than using a DDS chirp to control a voltage controlled oscillator (VCO) in a phase locked loop (PLL), particularly where the fractional bandwidth is moderate [9].

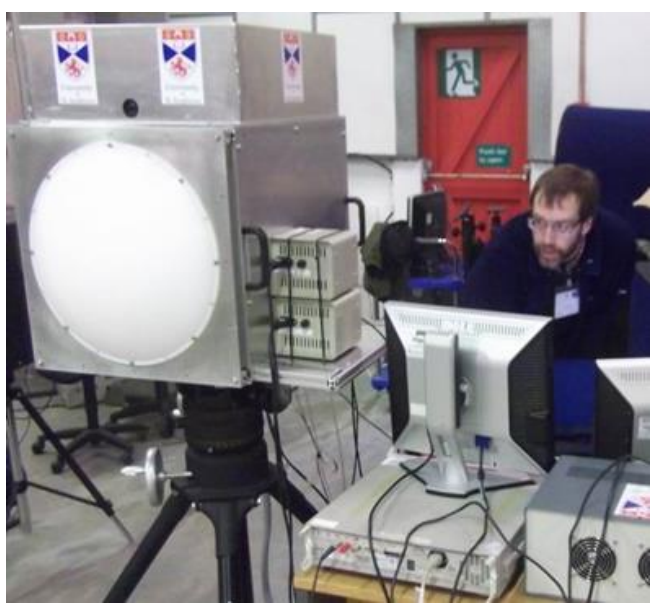

Figure 1: IRAD $340 \mathrm{GHz}$ radar undergoing field trials

A key design aspect of IRAD which enabled high frame rates was to use high speed, small diameter beam steering components prior to a two-lens telescope which provided the main focussing. We employed a conventional 2-axis galvanometer scanner which suited the long range design such that the $1.5^{\circ} \times 1.5^{\circ} \mathrm{FoV}$ was achieved with $\pm 14^{\circ}$ beam deflection ( $\pm 7^{\circ}$ galvo deflection) via the $\mathrm{M}=9$ telescope. A typical image size of $36 \times 36$ pixels requires 18 cycles of the fast axis mirror which at $10 \mathrm{~Hz}$ frame rate means driving the fast axis galvo at $180 \mathrm{~Hz}$ which is just within its maximum frequency of $200 \mathrm{~Hz}$. At frame rates above a few $\mathrm{Hz}$, the finite response characteristics of the galvo closed loop control circuits causes beam scan distortion but this is compensated by characterisation and pre-distortion of the driving waveform [11].

Transmit-receive duplexing and co- and cross-polar receive separation are achieved with a quasi-optical duplexer based on wire grid polarisers and a ferrite Faraday rotator [12,6]. This offers excellent performance in terms of low insertion loss and high isolation over wide bandwidths at the expense of physical size when compared with waveguide circuits. However, the overall radar size $(63 \times 74 \times 74 \mathrm{~cm})$ is dominated by the $45 \mathrm{~cm}$ diameter primary lens.

The final technological aspect of IRAD which enables high frame rates is a high speed FMCW radar signal processing software architecture which exploits parallel processing on multi-core CPUs and hardware graphics routines on running on GPU hardware $[6,11]$. The incoming data stream from the data acquisition hardware is buffered and parallelised between multiple processing threads (proportional to the number of CPU cores) which undertake FFTs on each 3D image frame. The resultant range profiles are transferred to the graphics card and are scaled and rendered as co- and cross-polar intensity images using OpenGL fragment shaders - this is handled by graphics hardware with no additional load 
on the CPU. The net result is a very fast and scalable processing architecture which can run at frame rates far faster than achievable with the beam steering mechanics. In the case of IRAD the $>50 \mathrm{MB} / \mathrm{s}$ data rate is easily handled on very modest PC hardware.

Threat detection with IRAD is performed by analysing polarisation signatures. In general, man-made objects scatter more in the cross-polar than do humans. Fig. 2 shows IRAD single range bin frames of a person with no threat and with two different threat objects.

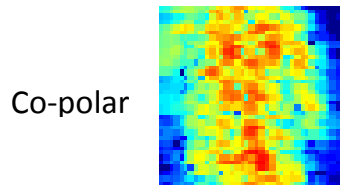

Blank

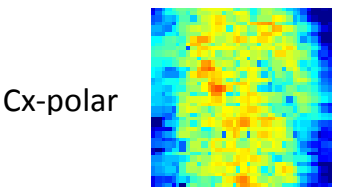

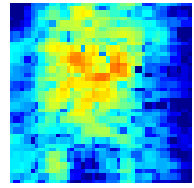

Threat 1

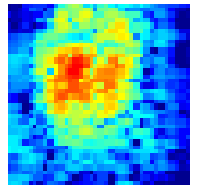

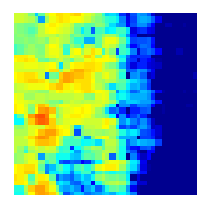

Threat 2

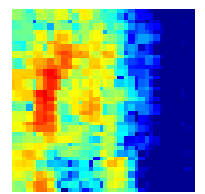

Figure 2: Example IRAD images: blank subject (left), subject with threat 1 (middle), subject with threat 2 (right).

We evaluated the threat detection performance of IRAD against a wide range of human subjects and various threat and benign items in a series of UK Home Office trials [13]. To achieve this we developed an algorithm which automatically detects, tracks and isolates a person in the search volume, then analyses their polarisation signature to derive an anomaly index which is related to the presence of a concealed object. The anomaly index is then used to classify the subject in real time. Statistical analysis of the large volume of trials data has enabled performance metrics for the radar system to be evaluated.

\section{Pathfinder $220 \mathrm{GHz}$ radar}

Within the EU FP7 CONSORTIS (Concealed Object StandOff Real-Time Imaging for Security) project, which aims to demonstrate high resolution, real time airport security screening with significantly improved throughput rates [14], we have constructed a $220 \mathrm{GHz}$ single pixel experimental radar called Pathfinder. This has been designed as a test bed for (i) novel transceiver technology from our project partner Wasa Millimetre Wave AB and (ii) our own wideband chirp generator architecture, both of which are candidate technologies for the future CONSORTIS security scanner. Additionally, Pathfinder has been useful as a data gathering platform to collect sub- $\mathrm{cm}^{3}$ volumetric resolution phenomenological data to aid the development of automatic anomaly detection algorithms by our project partner the Swedish Defence Agency FOI.
The transceiver uses MMIC and Schottky diode frequency multiplication (x24 in this case) and employs a novel selfmixing doubler output stage which operates in a balanced circuit configuration to act as both the final doubler on transmit and as homodyne subharmonic mixer on receive [15]. This offers the significant advantages of requiring no external duplexer (waveguide or quasi-optical), simpler hardware and the realistic prospect of being able to employ arrays of multiple transceivers in future systems. Whilst the self-mixing multiplier topology incurs slightly reduced performance ( $+3 \mathrm{dBm}$ output power, $-17 \mathrm{~dB}$ conversion loss) compared to having separate transmit and receive multiplier chains and a stand-alone mixer, the achievable performance is more than sufficient for the application and the advantages outweigh the disadvantages.

Pathfinder uses a single $\mathrm{x} 24$ self-mixing multiplier transceiver and a $22 \mathrm{~cm}$ diameter plano-convex HDPE lens which focusses the beam to a $\sim 1 \mathrm{~cm}$ spot at $\sim 1.5 \mathrm{~m}$ from the radar. To achieve the very high range resolution of $0.5 \mathrm{~cm}$ a chirp bandwidth of $30 \mathrm{GHz}$ is used (14\% fractional bandwidth). The chirp generator uses an upconverted DDS architecture to yield high speed, highly linear, low phase noise chirps at $\sim 8.9$ GHz. The whole assembly is mounted on an elevation-overazimuth gimbal which is used to raster the beam across the scene. Radar data is collected with a USB data acquisition card and processed offline in MATLAB. Pathfinder is shown in Fig. 3.

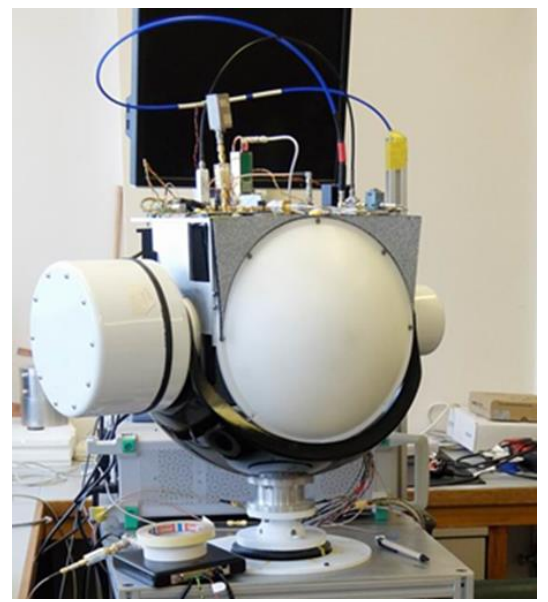

Figure 3: Pathfinder $220 \mathrm{GHz}$ radar.

The radar's point response was characterised by measuring the range profile to a point target located at the beam focus. The target was a miniature corner cube $(\sim 6 \times 6 \times 6 \mathrm{~mm})$, which is smaller than the $3 \mathrm{~dB}$ width of the beam, that has a large radar cross section and provides a high signal-to-noise ratio. Despite the very wide chirp bandwidth, the raw point response of Pathfinder is excellent with relatively little spectral spreading and quite low level range sidelobes. Previously reported results for wideband submillimetre wave radars have exhibited very messy raw point responses, broadened over many range bins and barely showing a distinct peak, which are only usable once compensation 
routines are applied to the data. In contrast, Pathfinder has a very clean raw point response with a distinct peak and range sidelobes no higher than $-30 \mathrm{dBc}$. Applying compensation yields a point response with transform-limited resolution over a dynamic range of nearly $70 \mathrm{~dB}$. The high quality raw point response is attributed to the highly linear chirp generator and the excellent amplitude flatness and group delay characteristics of the transceiver.

As a result of the excellent range resolution $(0.5 \mathrm{~cm}$ range bins) and cleanly focussed beam $(<1 \mathrm{~cm}$ spot $)$ with low sidelobes $(<-20 \mathrm{~dB}$ one-way), Pathfinder is capable of collecting high fidelity $3 \mathrm{D}$ data with sub $-\mathrm{cm}^{3}$ voxel resolution which resolves quite small spatial features whilst penetrating multiple layers of clothing. An example point cloud of a mannequin imaged with Pathfinder is shown in Fig. 4. Due to only having a single pixel and gimbal beam steering, Pathfinder is very slow - this image took five hours to acquire! However, as a technology test bed and a tool for gathering sub- $\mathrm{cm}^{3}$ voxel resolution data it has proven invaluable.

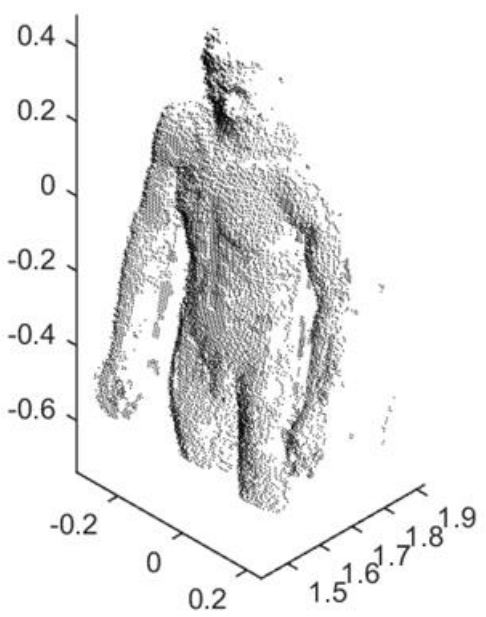

Figure 4: Pathfinder $220 \mathrm{GHz}$ point cloud of mannequin.

\section{CONSORTIS $340 \mathrm{GHz}$ radar}

The goal of the EU FP7 CONSORTIS project is to demonstrate high resolution, real time airport security screening with significantly improved throughput rates using submillimetre wave passive and active imaging technology. The project consortium is designing a system which will combine a dual-band passive submillimetre wave imager operating at 250 and $500 \mathrm{GHz}$ and an active 3D imaging radar operating at $340 \mathrm{GHz}$ as a technology demonstrator addressing future airport security requirements for walkthrough security lanes [16]. St Andrews is leading the design and development of the radar. Fig. 5 shows a CAD model of the CONSORTIS radar.

The CONSORTIS radar is designed to gather $1 \mathrm{~cm}^{3}$ voxel resolution 3D data over a $1 \times 1 \mathrm{~m}^{2} \mathrm{FoV}$ at $10 \mathrm{~Hz}$ frame rate at a range of a few metres. Achieving such a wide FoV and high frame rate is particularly demanding and has resulted in a number of advanced design aspects being selected. Key to achieving the desired frame rate is the use of a sparse linear focal plane array of 16 homodyne FMCW radar transceivers which use the same self-mixing multiplier technology proven in Pathfinder. The CONSORTIS transceivers will use x32 multiplication and be driven by a 16-way output DDS-based chirp generator based on the Pathfinder architecture to yield $30 \mathrm{GHz}$ chirps centred at $340 \mathrm{GHz}$. The transceivers are coupled to free space using smooth-walled spline feedhorns manufactured in E-plane split-block technology to minimise unit cost.

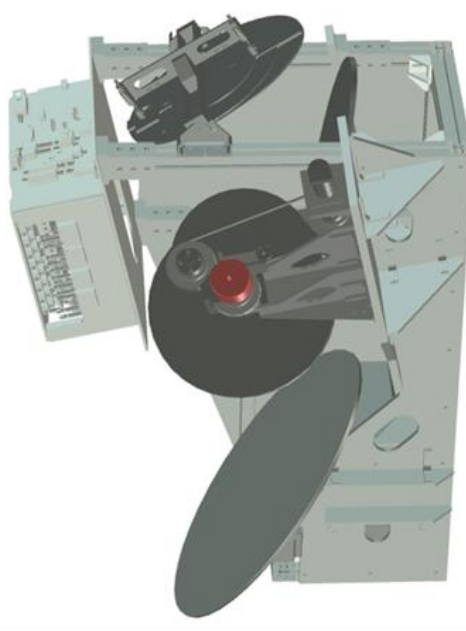

Figure 5: CAD model of CONSORTIS $340 \mathrm{GHz}$ radar. The 16 channel focal plane array of transceivers is housed in the box at upper left. The Dragonian mirror pair, double disc scanner and pan mirror are seen in the centre supported by the radar sub-chassis.

Achieving good beam quality across a wide FoV is particularly challenging when using a physically large focal plane array. Our project partner TUDelft has designed Dragonian mirror focussing optics which achieve a $1 \times 1 \mathrm{~m}^{2}$ FoV with $1 \mathrm{~m}$ depth of field and a $1 \mathrm{~cm}$ central spot size whilst maintaining low aberrations and scan loss [17]. The 16 transceiver beams are rastered across the FoV using optomechanical beam scanning. A double-disc scanner performs a fast axis linear scan perpendicular to the array direction whilst the Dragonian primary performs a slow axis reciprocation to fill in the sampling pattern.

Finally, there are nearly 15 million voxels in every $100 \mathrm{~ms}$ frame in the imaging volume which need to be processed in real time, we have developed an enhanced version of the hybrid CPU/GPU processing code originally written for IRAD to cope with the demanding data rate. For example, whilst the original IRAD code would have processed and rendered this data rate at only $\sim 1 \mathrm{~Hz}$, the enhanced code will achieve a frame rate of $>30 \mathrm{~Hz}$, which far exceeds the mechanical beam scanning limit. 
With these advanced design features, we believe the CONSORTIS radar will set new standards of performance for submillimetre wave 3D imaging radar.

\section{Conclusions}

Submillimetre wave 3D imaging radar continues to develop as a technology suitable for short and long range security applications. The work currently being undertaken at $\mathrm{St}$ Andrews, in conjunction with collaborators, to develop the CONSORTIS $340 \mathrm{GHz}$ radar builds on our prior work and aims to combine the multiple goals of wide field of view, high voxel resolution and high frame rate. Future challenges for the field will be to increase further the pixel count, field of view and frame rate. Bigger arrays of transceivers will be achieved through greater component level integration and the move towards submillimetre wave MMICs. Successful application of submillimetre wave security radars will also require substantial effort in developing robust and high speed automatic image analysis software.

\section{Acknowledgements}

A proportion of this work was carried out as part of the UK MOD's Electronic Systems Research Programme. Part of the research leading to these results has received funding from the European Union Seventh Framework Programme (FP7/2007-2013) under grant agreement no. 312745. The authors also wish to acknowledge funding which has supported this work from the UK Natural Environment Research Council and the UK Home Office Centre for Applied Science \& Technology. They are grateful to the many colleagues and collaborators who have contributed to making these projects successful.

\section{References}

[1] Appleby, R. \& Wallace, H.B., "Standoff detection of weapons and contraband in the $100 \mathrm{GHz}$ to $1 \mathrm{THz}$ region," IEEE Trans. Antennas Propag., vol. 55, no. 11, pp. 2944-2956, (2007).

[2] L3 Security \& Detection Systems Advanced Personnel Screening website: http://www.sds.l$\underline{3 \text { com.com/products/advancedimagingtech.htm }}$

[3] Cooper, K.B. \& Chattopadhyay, G., "SubmillimeterWave Radar: Solid-State System Design and Applications", IEEE Microwave Magazine, vol. 15, no. 7, pp. 51-67, (2014).

[4] Cooper, K.B., et al., "THz Imaging Radar for Standoff Personnel Screening," IEEE Trans. Terahertz Science and Technology, vol. 1, no. 1, pp. 169 - 182, (2011)

[5] Sheen, D.M., Hall, T.E., Severtsen, R.H, McMakin, D.L., Hatchell, B.K. and Valdez, P.L.J., "Standoff concealed weapon detection using a $350 \mathrm{GHz}$ radar imaging system", Proc. SPIE 7670, Passive MillimeterWave Imaging Technology XIII, 767008, (2011).

[6] Robertson, D.A., Marsh, P.N., Bolton, D.R., Middleton, R.J.C., Hunter, R.I., Speirs, P.J., Macfarlane, D.G.,
Cassidy, S.L., and Smith, G.M., "340 GHz 3D radar imaging test bed with $10 \mathrm{~Hz}$ frame rate.", Proc. SPIE 8362, Passive and Active Millimeter-Wave Imaging XV, 836206, (2012).

[7] Grajal, J.; Badolato, A.; Rubio-Cidre, G.; UbedaMedina, L.; Mencia-Oliva, B.; Garcia-Pino, A.; Gonzalez-Valdes, B.; Rubinos, O, “ 3-D HighResolution Imaging Radar at $300 \mathrm{GHz}$ With Enhanced FoV", IEEE Trans. Microwave Theory Tech., vol. 63, no. 3, pp. 1097-1107, (2015).

[8] Deal, W.R., "InP HEMT for Sub-Millimeter Wave Space Applications: Status and Challenges", Int. Conf. Infrared, Millimeter and Terahertz Waves (IRMMWTHz), pp. 1- 3, (2014).

[9] Robertson, D.A., Cassidy, S.L. \& Bolton, D.R., "Nonlinearity and phase noise effects in $340 \mathrm{GHz}$ 3D imaging radar", Proc. SPIE 8715, Passive and Active Millimeter-Wave Imaging XVI, 87150M, (2013).

[10] Cooper, K.B., Trabert, J.F. \& Dengler, R.J., "Carrier Noise-Limited Penetration in $\mathrm{THz}$ Radar Imaging", IEEE MTT-S International Microwave Symposium Digest (MTT), pp. 1-3, (2012).

[11] Cassidy, S.L. "Millimetre-wave FMCW radar for remote sensing and security applications", PhD Thesis, University of St Andrews, (2015).

[12] Hunter, R.I., Robertson, D.A., Goy, P. and Smith, G.M., "Design of High-Performance Millimeter Wave and Sub-Millimeter Wave Quasi-Optical Isolators and Circulators", IEEE. Trans. Microwave Theory Tech., vol. 55, no. 5, pp. 890 - 898, (2007).

[13] Robertson, D.A., Cassidy, S.L., Jones, B. \& Clark A., "Concealed threat detection with the IRAD submillimeter wave 3D imaging radar", Proc. SPIE. 9078, Passive and Active Millimeter-Wave Imaging XVII, 907805. (2014).

[14] CONSORTIS website: http://consortis.eu/

[15] Bryllert, T., Drakinskiy, V., Cooper, K.B., \& Stake, J., "Integrated 200-240-GHz FMCW Radar Transceiver Module", IEEE Trans. Microwave Theory Tech., vol. 61, no. 10, pp. 3808 - 3815, (2013).

[16] Appleby, R. Petersson, H. \& Ferguson, S., "Concealed object stand-off real-time imaging for security: CONSORTIS", Proc. SPIE. 9462, Passive and Active Millimeter-Wave Imaging XVIII, 946204, (2015).

[17] Gandini, E. \& Llombart, N., "Toward a real time standoff submillimeter-wave imaging system with large field of view: quasi-optical system design considerations", Proc. SPIE 9462, Passive and Active Millimeter-Wave Imaging XVIII, 946205, (2015). 